\title{
¿Qué Opinan los Maestros sobre las Competencias de Liderazgo Escolar y sobre su Formación Inicial?
}

\author{
What do Teachers Think about School Leadership Competences \\ and their Pre-Service Training?
}

\author{
Pilar Iranzo-García * \\ Marta Camarero-Figuerola \\ Charo Barrios-Arós \\ Juana-María Tierno-García \\ Sandra Gilabert-Medina \\ Universidad Rovira i Virgili, España
}

\begin{abstract}
Las competencias para el liderazgo escolar en la formación inicial de los maestros son necesarias porque la acción docente en las aulas se tiene que aliar con el liderazgo y la organización óptima del centro educativo y ello comporta competencias para la coordinación y la colaboración con el entorno. Este artículo se centra en la visión sobre dichas competencias que tienen los maestros y equipos directivos que colaboran en las prácticas externas de los estudiantes. Para ello se realiza un estudio de casos múltiple con metodología mixta, con la participación de tres escuelas de educación infantil y primaria. La muestra de estudio $(n=20)$ la configuran equipos directivos y maestros vinculados a Educación infantil y ciclo inicial de Educación primaria. El instrumento utilizado es un cuestionario ad hoc, distribuido online durante el curso 2016-2017. Los maestros y directores perciben que, del conjunto de las funciones docentes, la coordinación es la más deficitaria, aunque la más importante, por encima del liderazgo y la colaboración con el entorno. Los profesores consideran mejorables algunas competencias relacionadas con el liderazgo como resolución de conflictos, análisis de situaciones complejas, gestión del aula y, por último, relación con familias y entidades del entorno.
\end{abstract}

Descriptores: Liderazgo; Coordinación de la educación; Docente; Competencias del docente; Formación de docentes.

Competencies for school leadership in the initial training of teachers are necessary because the teaching action in the classrooms must be allied with leadership and the optimal organization of the educational institution and this includes competences for coordination and networking with the community. This article focuses on the views on these competencies of teaching staff and school leaders who collaborate on the practicum stage of students. To this end, a multiple case study is conducted using mixed methodology, with the participation of three pre-school and primary schools. The study sample $(n=20)$ is made up of members of management teams and teachers who are working in pre-school or the two first years of primary school. The instrument used is an ad hoc questionnaire, distributed online during the 2016-2017 academic year. The results show that teachers and principals perceive that, from all the teaching functions, coordination is the less developed although it is the most important, above leadership and the relationship with the socio-educational environment. Teachers, they consider that the following competencies, related to leadership, can be improved: conflict resolution, complex situations analysis, classroom management and relationship with families and entities in the educational context.

Keywords: Leadership; Educational coordination; Teachers; Teacher qualifications; Teacher education.

*Contacto: pilar.iranzo@urv.cat

ISSN: $1696-4713$

www.rinace.net/reice/

revistas.uam.es/reice
Recibido: $\quad 21$ de enero 2018

$1^{\text {a }}$ Evaluación: 17 de marzo 2018

$2^{\text {a }}$ Evaluación: 30 de abril 2018

Aceptado: $\quad 27$ de mayo 2018 


\section{Introducción}

La formación inicial de los maestros pretende la construcción de una identidad docente activa capaz de comprometerse con la mejora educativa y social, el desarrollo de conocimientos psicopedagógicos relevantes y la dotación de competencias para la participación en las culturas organizativas de los centros en los que ejercerán su profesión. Para que los estudiantes inicien el proceso de primera inserción a la profesión se requiere, obviamente, de tutorizaciones y acompañamientos exhaustivos y ricos en interacciones profesionalizadoras por parte del profesorado universitario y de los maestros y tutores de los centros escolares (Agudo, 2017; Cano, 2011; Molina, 2004; Negrillo e Iranzo-García, 2009; Putnam y Borko, 2000).

En general, en las culturas escolares tendentes al individualismo la identidad docente se construiría atendiendo a funciones sobre todo vinculadas con las situaciones de enseñanzaaprendizaje en el escenario "aula" (Hargreaves, 1996). Pero hemos aprendido que la inclusión, la mejora escolar y la justicia social requieren enfatizar en 'todos' los docentes dimensiones de la identidad profesional vinculadas a los escenarios "centro" y "comunidad" para desarrollar los liderazgos pedagógico, distribuido y contextualizado (Bolívar, 2010, 2011; Iranzo-García, Tierno-García y Barrios-Arós, 2014). Además, el desarrollo legal y la trayectoria de profesionalización realizados en España y Cataluña en los últimos tiempos, lleva pareja la necesidad de una formación inicial y permanente para la función directiva que asegure una continuada capacitación para la mejora escolar compartida entre toda la comunidad (Iranzo-García et al., 2018).

En este sentido, interesa indagar en cómo se construyen las competencias docentes referidas a la coordinación interna, el coliderazgo y el trabajo en red con la comunidad a lo largo de la formación inicial, en general, y durante las prácticas externas, en particular.

\section{Revisión de la literatura}

Centramos la revisión de la literatura fundamentalmente en los ámbitos del estado del liderazgo en la profesión docente y en cómo la formación inicial de los maestros debe abordar las competencias de liderazgo escolar. Ambos tópicos se centran preferentemente en el contexto español porque interesa conocer cómo se desarrollan los mismos en un sistema escolar que tiene semiprofesionalizada la función de la dirección y, en consecuencia, una débil definición del liderazgo escolar en España que tiene su correlato en cómo se aborda éste en la formación inicial de los maestros.

En lo relativo al liderazgo escolar, si bien se acepta internacionalmente que deben confluir los liderazgos pedagógico, distribuido y contextualizado para que los centros educativos sean inclusivos y como tales lugares dónde toda la comunidad aprende, decide y compensa desigualdades provenientes de círculos de desventaja, todavía es un reto que eso suceda en la práctica (Iranzo-García, Tierno-García y Barrios-Arós, 20 14; León, Crisol y Moreno, 2018; OCDE, 2009).

La cultura escolar española se ha caracterizado hasta épocas recientes por un debate lentamente orientado hacia la profesionalización de la función de liderazgo escolar y, en consecuencia, por una débil política educativa centrada en la capacitación de los directoresas para desarrollar ese triple y complejo liderazgo del que hablamos. Resultados concretos de todo ello han sido, en parte, una cierta 'evitación’ de acceder a la función directiva en el 
conjunto de España con algunas diferencias en sus diversas autonomías (Aramendi, Teixidó y Bernal, 2010; Barrios-Arós, Iranzo-García y Tierno-García, 2013) e identidades directivas más vinculadas a la docencia o de primus inter pares (Cantón y Arias, 2008; Gairín y Castro, 2010).

Lo cierto es que si bien el liderazgo distribuido tiene ya un indiscutible asentamiento teórico en los sistemas educativos internacionales (Danielson, 2006; Leithwood, 2009; Leithwood et al., 2006; Murillo, 2006; OCDE, 2009; Spillane, 2006;), se reconoce que, en la práctica, las estructuras organizativas escolares son lugares complejos en los que aprender a liderar de forma colaborativa requiere competencias comprensivas, estratégicas y actitudinales. Como recogíamos en Iranzo-García, Tierno-García y Barrios-Arós (2014), las competencias para la función directiva serían:

- Identificar y establecer políticas y finalidades del centro, es decir, marcar dirección a partir de la autoevaluación institucional colegiada.

- Focalizar la atención sobre enseñanza y aprendizaje de toda la comunidad.

- Establecer un corpus de relaciones interpersonales que aumenten la participación dentro y fuera de la comunidad educativa y permitan redes de colaboración, trabajo en equipo y coliderazgo en el seno de la institución.

- Fortalecer relaciones externas con el entorno más amplio.

En esa misma fuente se evidenciaba que la autoevaluación institucional colegiada, la coordinación interna y las relaciones escuela-familias y el trabajo en red con la comunidad eran ámbitos en los que los equipos directivos participantes en la investigación mostraban dificultades.

En relación a la formación inicial de maestros, los resultados de investigaciones recientes como "El impacto de la evaluación educativa en el desarrollo de competencias en la universidad. La perspectiva de las primeras promocionas de graduados" (Cano, 2013) ponen de manifiesto, concretamente en el Grado de Educación Primaria, que, cada vez más, los colectivos implicados en la Educación Superior (profesores, egresados, coordinadores académicos y empleadores) destacan la importancia de competencias transversales que atenderían a la capacitación para asumir funciones vinculadas globalmente a la organización escolar y no sólo a los procesos de enseñanza-aprendizaje desde competencias didáctico-curriculares. Se resaltan especialmente, y por este orden:

- El trabajo en equipo, trabajo colaborativo o cooperativo.

- La competencia comunicativa en sentido amplio: comunicación y habilitados sociales y personales.

- La resolución de problemas o de situaciones complejas en las que no es suficiente aplicar una serie de pasos preestablecidos o reglas cerradas, sino que implican juicio crítico y pensamiento creativo e innovador.

- El aprendizaje autónomo, que incluye las estrategias de autorregulación.

El estudio también revela que hay otras competencias que a pesar de considerarse muy importantes (compromiso ético, iniciativa, emprendeduría, liderazgo de proyectos y relación con el entorno) no se abordarían suficientemente en la formación de los maestros debido a, entre otras razones, la prevalencia de otras más instrumentales, más simples o tradicionalmente más presentes. 
Estos resultados son congruentes con los obtenidos en el marco del "proyecto empleadores" (AQU Catalunya, 2015a) que pretende aportar la visión de las empresas y las instituciones sobre la ocupabilidad de la población graduada recientemente. En el ámbito educativo, una de las principales conclusiones es que la satisfacción con las competencias de los nuevos docentes es aceptable (6,8 sobre 10) pero, ciertamente, con un margen de mejora importante, especialmente en relación a los centros de más complejidad.

Por este motivo, una de las propuestas de ese informe es promover una visión holística de la formación inicial y continua de los docentes, que permita el logro y la actualización continuada de las competencias disciplinares, pero también de las transversales: gestión del aula y de conflictos, responsabilidad en el trabajo, promoción de valores y respeto, entrevistas con las familias, trabajo colaborativo o liderazgo, entre otros (AQU Catalunya, 2015b).

En línea también con nuestros argumentos, Perrenoud (2007) afirma que en la formación inicial de los futuros maestros es fundamental incluir competencias como las de:

- Elaborar un proyecto de equipo y construir representaciones comunes.

- Impulsar un grupo de trabajo y dirigir reuniones.

- Formar y renovar un equipo pedagógico.

- Confrontar situaciones complejas, prácticas y problemas profesionales y analizarlos conjuntamente.

- Hacer frente a crisis o conflictos entre personas.

En respuesta a todo lo planteado anteriormente, nuestro proyecto (ARMIF-2015) pretende analizar cómo se están construyendo competencias vinculadas al liderazgo, coordinación interna y relación con la comunidad por parte de varios agentes implicados en la calidad de la profesión docente y la formación inicial de maestros (profesorado y responsables académicos universitarios, estudiantes del Grado de Educación Infantil de la Universitat Rovira i Virgili (URV) y maestras y directores de centros educativos de la zona que tutorizan estudiantes en prácticas de la URV. El análisis incluye el contraste de cómo se estarían construyendo en otra universidad del contex to español (Granada) y la perspectiva internacional sobre el foco de estudio.

Este artículo tiene el propósito de mostrar la visión que tienen los maestros y los equipos directivos de las tres escuelas participantes, como forma de aproximación al modelo de liderazgo que se está desarrollando en los centros de prácticas y a la consiguiente influencia que tales modelos pueden ejercer en la construcción de competencias de coordinación, liderazgo y relación con el entorno por parte de los estudiantes de maestroa. Concretamente nos centramos en su conceptualización, su importancia, el impacto que tienen en el ejercicio docente y el estado actual de las mismas en sus respectivos centros escolares, además de analizar su percepción sobre el nivel competencial de los estudiantes en prácticas y del profesorado novel.

\section{Método}

El diseño de la investigación se basa en un estudio de casos múltiple con metodología mixta. Para ello se cuenta con la participación de tres centros escolares públicos de educación infantil y primaria de la provincia de Tarragona no catalogados como centros 
de máxima complejidad (Generalitat de Catalunya, 2014). Se han seleccionado de manera intencional, atendiendo a los siguientes criterios: acogen regularmente a estudiantes en prácticas del Grado de Educación Infantil y Primaria y, además, el equipo directivo ha mostrado interés por participar en el proyecto.

La muestra de estudio la configuran los equipos directivos de las escuelas y los maestros vinculados a la etapa de educación infantil y ciclo inicial de educación primaria $(\mathrm{n}=20)$. A continuación, detallamos la composición de esta muestra en lo referido a sus funciones unipersonales y colegiadas. Interesa resaltar que, en general, la docencia está ampliamente compartida con funciones de coordinación o complementarias (cuadro 1). Si se atiende a otras variables, la caracterización de la muestra aparece en el cuadro 2.

Cuadro 1. Distribución de la muestra por escuelas y cargos ejercidos

\begin{tabular}{|c|c|c|c|c|c|}
\hline ID & ESCUELA A & ID & ESCUELA B & ID & Escuela C \\
\hline $\mathrm{DA} 1$ & Directora & $\mathrm{DB} 1$ & Director & $\mathrm{DC} 1$ & $\begin{array}{l}\text { Director y tutor de } \\
6^{\circ}\end{array}$ \\
\hline JA1 & Jefa de estudios & $\mathrm{JB} 1$ & Jefa de Estudios & $\mathrm{MC} 1$ & Maestra de apoyo \\
\hline $\mathrm{SA} 1$ & $\begin{array}{l}\text { Secretaria + tutora de } 6^{\circ} \\
+ \text { coordinadora de ciclo } \\
\text { superior }\end{array}$ & $\mathrm{SB} 1$ & Secretario & $\mathrm{CC} 1$ & $\begin{array}{l}\text { Tutora P5 y } \\
\text { coordinadora de } \\
\text { Ciclo Infantil }\end{array}$ \\
\hline $\mathrm{CA} 1$ & $\begin{array}{l}\text { Coordinadora Biblioteca } \\
+ \text { tutora de segundo }\end{array}$ & $\mathrm{CB} 1$ & $\begin{array}{l}\text { Coordinadora Ciclo } \\
\text { Infantil y tutora } \mathrm{P}_{5}\end{array}$ & $\mathrm{TC} 1$ & Tutora $\mathrm{P} 4$ \\
\hline $\mathrm{CA} 2$ & $\begin{array}{l}\text { Coordinadora Ciclo } \\
\text { Inicial }\end{array}$ & $\mathrm{TB} 1$ & Tutora P5 & $\mathrm{TC} 2$ & $\begin{array}{l}\text { Tutora P3 y } \\
\text { Maestra Educación } \\
\text { Especial }\end{array}$ \\
\hline CA3 & $\begin{array}{l}\text { Tutor P3 y Coordinador } \\
\text { TIC-TAC }\end{array}$ & $\mathrm{TB} 2$ & Tutora P4 & & \\
\hline $\mathrm{CA} 4$ & $\begin{array}{l}\text { Tutora } \mathrm{P} 4 \mathrm{y} \\
\text { Coordinadora Ciclo } \\
\text { Infantil }\end{array}$ & TB3 & Tutora P3 & & \\
\hline TA1 & Tutora P5 & & & & \\
\hline
\end{tabular}

Fuente: Elaboración propia.

Como se ve, se trataría de un colectivo equilibrado respecto a la edad, experimentado y mayormente estable laboral y profesionalmente. Se percibe también la tradicional feminización docente de la etapa educativa infantil (Eurydice, 2013).

El instrumento utilizado es un cuestionario ad hoc, diseñado a partir de un análisis bibliográfico y distribuido a través de la plataforma online Lime Survey durante el curso académico 2016-2017. El cuestionario ha sido validado por expertos nacionales e internacionales, y también mediante una prueba piloto con los tres directores de las escuelas participantes. Fruto de estas revisiones se redujo el número de preguntas, se adecuaron las nomenclaturas al vocabulario utilizado por los docentes y se ampliaron las opciones de respuesta.

La versión definitiva del cuestionario está compuesta por 25 preguntas abiertas y cerradas de selección múltiple y de escala. El cuestionario se estructura en dos bloques:

- Datos de identificación del centro y del docente: nombre de la escuela; edad; género; formación inicial y especialidad; ciclos donde se imparte la docencia; situación profesional; dedicación; total de años de experiencia docente, cargos y funciones desempeñados y experiencia en tutorización de prácticas del alumnado universitario. 
- Percepción de las competencias de liderazgo: selección de las competencias más relevantes del docente; autovaloración del nivel competencial; atribución del nivel competencial; necesidades formativas; aspectos y competencias más influyentes en el ejercicio docente; definiciones y autovaloración de "coordinación", "liderazgo", "liderazgo compartido" y "colaboración con el entorno"; Percepción del nivel competencial de los maestros noveles y/o estudiantes en prácticas.

Cuadro 2. Perfil de la muestra de maestros y equipos directivos de las 3 escuelas

\begin{tabular}{|c|c|c|c|c|}
\hline VARIABLES & ESCUELA A & ESCUELA B & Escuela C & TOTAL \\
\hline Género & $\begin{array}{l}87,5 \% \text { Mujeres } \\
12,5 \% \text { Hombres }\end{array}$ & $\begin{array}{l}71,4 \% \text { Mujeres } \\
28,6 \% \text { Hombres }\end{array}$ & $\begin{array}{l}80 \% \text { Mujeres } \\
20 \% \text { Hombres }\end{array}$ & $\begin{array}{l}80 \% \text { mujeres } \\
20 \% \text { hombres }\end{array}$ \\
\hline Edad & $\begin{array}{l}62,5 \%<40 \text { años } \\
37,5 \% \geq 40 \text { años }\end{array}$ & $\begin{array}{l}28,6 \%<40 \text { años } \\
71,4 \% \geq 40 \text { años }\end{array}$ & $\begin{array}{l}40 \%<40 \text { años } \\
60 \% \geq 40 \text { años } \\
\end{array}$ & $\begin{array}{l}50 \%<40 \text { años } \\
50 \% \geq 40 \text { años }\end{array}$ \\
\hline $\begin{array}{l}\text { Años de } \\
\text { experiencia } \\
\text { docente }\end{array}$ & $\begin{array}{l}37,5 \%<11 \text { años } \\
62,5 \% \geq 11 \text { años }\end{array}$ & $\begin{array}{l}14,3 \%<11 \text { años } \\
85,7 \% \geq 11 \text { años }\end{array}$ & $\begin{array}{l}60 \%<11 \text { años } \\
40 \% \geq 11 \text { años }\end{array}$ & $\begin{array}{l}30 \%<11 \text { años } \\
70 \% \geq 11 \text { años }\end{array}$ \\
\hline Tipo de plaza & $\begin{array}{l}50 \% \text { Definitiva } \\
50 \% \text { Provisional }\end{array}$ & $\begin{array}{l}85,7 \% \text { Definitiva } \\
14,3 \% \text { Provisional }\end{array}$ & $\begin{array}{l}60 \% \text { Definitiva } \\
40 \% \\
\text { Provisional }\end{array}$ & $\begin{array}{l}65 \% \text { definitiva } \\
35 \% \text { provisional }\end{array}$ \\
\hline $\begin{array}{l}\text { Puesto de } \\
\text { trabajo } \\
\text { específico }^{1}\end{array}$ & $\begin{array}{l}25 \% \text { Sí } \\
75 \% \text { No }\end{array}$ & $\begin{array}{l}\text { 14,30\% Sí } \\
85,7 \% \mathrm{No}\end{array}$ & $100 \%$ No & $\begin{array}{l}15 \% \text { Sí } \\
85 \% \text { No }\end{array}$ \\
\hline $\begin{array}{l}\text { Tutorización } \\
\text { de prácticas } \\
\end{array}$ & $\begin{array}{l}75 \% \text { Sí } \\
25 \% \text { No } \\
\end{array}$ & $\begin{array}{l}71,4 \% \text { Sí } \\
28,6 \% \text { No } \\
\end{array}$ & $\begin{array}{l}80 \% \text { Sí } \\
20 \% \text { No } \\
\end{array}$ & $\begin{array}{l}75 \% \text { Sí } \\
25 \% \text { No }\end{array}$ \\
\hline Dedicación & $100 \%$ Completa & & & $100 \%$ Completa \\
\hline
\end{tabular}

* Realizada en los últimos tres años.

Fuente: Elaboración propia.

Para el tratamiento cualitativo de las preguntas abiertas se ha utilizado el análisis de contenido con objeto de identificar las principales categorías emergentes de la conceptualización de cada competencia (coordinación, liderazgo, colaboración con el entorno). En cuanto al análisis cuantitativo se ha realizado con el programa SPSS (v.22.0) mediante estadística descriptiva. Por último, la fiabilidad de los ítems del cuestionario se ha calculado mediante Alfa de Cronbach obteniendo un valor de 0,861.

\section{Resultados}

Presentamos los referidos a:

- ¿Cómo definen maestros y equipos directivos las competencias de coordinación, liderazgo y colaboración con el entorno?

\footnotetext{
${ }^{1}$ El decreto 38/2014, de 25 de marzo, regula en Cataluña los procedimientos para definir el perfil y la provisión de puestos de trabajo específicos. El equipo directivo, de acuerdo con el proyecto educativo del centro y el proyecto de dirección puede exigir, para seleccionar parte de la plantilla docente, requisitos adicionales a la titulación, formación, experiencia u otros que determinen el puesto de trabajo.
} 
- ¿Cómo ordenan estas competencias según su impacto en el ejercicio docente y cómo valoran su importancia y estado actual de las mismas en la profesión docente y en el propio centro educativo?

- ¿Cómo perciben el nivel competencial con el que llegan a la escuela los estudiantes en prácticas y los maestros noveles?

Se indican, además, algunas de las tendencias observadas más significativas para nuestro objeto de estudio atendiendo a características personales y profesionales de los docentes consultados como la edad, el género, la experiencia docente y la experiencia en la tutorización de estudiantes en prácticas.

\subsection{Definición de las competencias de coordinación, liderazgo y colaboración con el entorno}

En este apartado, les pedimos que definan con sus propias palabras (pregunta abierta) esas competencias. Una vez analizadas las respuestas, se establecieron categorías emergentes para cada competencia.

\subsubsection{Coordinación}

En la conceptualización de coordinación hemos identificado las categorías emergentes que aparecen en el cuadro 3.

Cuadro 3. Categorización del concepto de coordinación

\begin{tabular}{lc}
\hline CATEGORÍAS EMERGENTES & $\mathbf{N}^{\mathbf{0}}$ DE CITAS \\
\hline Evitar incongruencias & 5 \\
Acuerdos consensuados en el proceso & 4 \\
Trabajo en equipo & 4 \\
Colaboración con la comunidad & 3 \\
Co-liderazgo & 3 \\
Intercambio de conocimientos & 2 \\
\hline Total de citas & 21 \\
\hline
\end{tabular}

Fuente: Elaboración propia.

Este concepto aparece lleno de matices que podríamos sintetizar diciendo que, fundamentalmente, es entendido como la capacidad de llegar a acuerdos consensuados evitando incongruencias,

Hemos de ir por el mismo camino en aspectos, sobre todo, generales y hay que llegar a acuerdos y dialogar mucho. Encontrar puntos en común. (TA1)

...es importante que esta coordinación se establezca en un marco de línea metodológica (...) para ir todos en la misma dirección y con las ideas pautadas y claras. (DA1)

El trabajo en equipo se percibe como la estrategia para llegar a esos acuerdos, ya que la coordinación "implica el encuentro sistemático de un grupo de maestros para consensuar, programar, evaluar, hacer el seguimiento, ... de las tareas que tienen encargadas y de las que se propongan. (TB2)

Así mismo se reconoce la necesidad de un liderazgo en los distintos equipos de trabajo "podernos agrupar para trabajar en común algunos aspectos. Con la necesidad que uno de los participantes coordine y priorice tareas o temas que necesitamos trabajar. (TC1) 
... implica que la persona encargada de coordinar también ha de presentar un buen liderazgo, con capacidades y habilidades suficientes para motivar al equipo (...) no se puede limitar a un traspaso de información y ya está. (TC2)

En ese sentido, algunos docentes entienden que la colaboración con la comunidad y el intercambio de conocimientos es consustancial a la coordinación: "estar toda la comunidad educativa coordinada tanto en el funcionamiento como en la pedagogía y maneras de hacer y de pensar. (CC1)

toda la comunidad educativa ha de participar en la toma de decisiones, en la línea de trabajo del curso escolar, ser conocedora de las actividades y de todo lo que se haga en el centro y que esto surja de una decisión y programación conjunta. (MC1)

\subsubsection{Liderazgo}

Las categorías que han emergido sobre esta competencia aparecen en el cuadro 4.

Cuadro 4. Categorización del concepto de liderazgo

\begin{tabular}{lc}
\hline \multicolumn{1}{c}{ CATEGORÍAS EMERGENTES } & N $^{\circ}$ DE CITAS \\
\hline Función de marcar la dirección & 10 \\
Competencias personales del liderazgo & 6 \\
Función de motivar e implicar & 6 \\
\hline Total de citas & 22 \\
\hline
\end{tabular}

Fuente: Elaboración propia.

En la conceptualización de liderazgo destaca la idea de marcar una dirección, entendiendo el líder como,

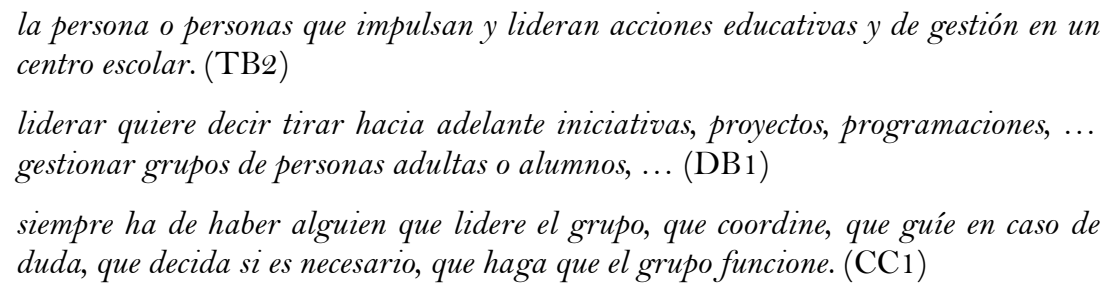

Sin embargo, en la mayoría de casos, este hecho se matiza con la función de motivar, impulsar, implicar al colectivo y no tanto desde una óptica impositiva "(Liderar) implica no imponer, saber escuchar, razonar de una forma convincente y asertiva, consensuar, pactar,... nunca imponiendo criterios ni actuaciones (DA1)”, “... saber explicar y exponer metodologías o tareas a realizar y que el resto se anime a seguirlo (JA1)” además de asumir algunas responsabilidades como "crear y favorecer las condiciones, el ambiente y los recursos para que el equipo docente pueda aportar el máximo de experiencia y saberes que mejoren los procesos de aprendizaje y relación en la escuela de todos sus miembros (DC1)".

Se entiende que se necesiten unas competencias personales específicas "suelen ser personas muy asertivas, respetuosas con las opiniones de los otros, (...) que tienen un gran valor en la comunidad educativa (TC2)", "han de tomar decisiones, tener iniciativa y escuchar todas las voces. También ha de ser lo más justo posible (TA1)”. Aparecen también múltiples referencias a la importancia al liderazgo de los maestros en el aula porque,

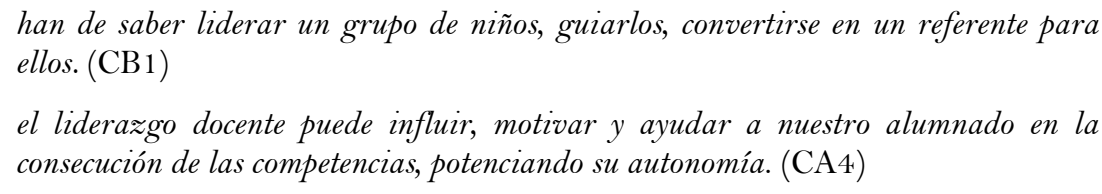


liderar un grupo-clase es mostrar los objetivos comunes a conseguir como un grupo humano que aprende, crece, se relaciona y convive de forma conjunta (...) se necesita una hoja de ruta, un proyecto que ha de ser coherente con el proyecto de centro. (SA1)

Desde esta idea de liderazgo, cuando se les pregunta en qué situaciones entienden que se ejerce el liderazgo en el ámbito del ejercicio docente, es obvio que la respuesta mayoritaria tienda a asumir que "a toda hora y en todas las situaciones (SB1)", "en casi todas las situaciones entre docentes, en el aula, en relación con las familias, ... (TC1)". Esa respuesta generalizada se concreta con elementos que conceden una especial importancia a la relación personal:

\section{En todo momento hay que mostrarse como un líder en el aula. Eso no quiere decir ser el protagonista, sino mostrar fuerza, ternura y alegría suficientes para guiar a los alumnos en su aprendizaje. (CB1) \\ El auténtico liderazgo docente en las aulas se ha de ejercer en los aspectos emocionales y más personales de los alumnos, allí donde los progresos tecnológicos no pueden sustituir la atención personalizada. (SA1)}

Un concepto relacionado con el anterior es el de liderazgo compartido (cuadro 5), diferente del concepto genérico de liderazgo.

Cuadro 5. Categorización del concepto de liderazgo compartido

\begin{tabular}{lc}
\hline CATEGORÍAS EMERGENTES & No DE CITAS $^{\mathbf{2}}$ \\
\hline Liderazgo distribuido & 10 \\
Fruto de la negociación y el consenso con la comunidad & 4 \\
Co-responsabilidad & 3 \\
Fruto de la coordinación & 2 \\
\hline Total de citas & 19 \\
\hline
\end{tabular}

Fuente: Elaboración propia.

Se identifica fundamentalmente con la idea de liderazgo distribuido “...que no recae sólo en una persona (CC1)", "el que no sólo es del equipo directivo sino el que se comparte con el resto de profesorado (CA2)". Esta idea, además, introduce la comunidad educativa en el ejercicio de liderazgo a partir de procesos de negociación y consenso "... es hacer red, hacer comunidad, establecer complicidades, (...) construir relaciones con personas y grupos promoviendo constantemente la interacción y resolución de problemas (MC1)", "es el que da importancia a la gestión colectiva con una participación democrática y fomenta el trabajo coordinado en red (TB2)". Así mismo, se amplía el concepto con las condiciones de coordinación "es necesario el liderazgo compartido siempre y cuando estén las tareas distribuidas de forma coherente y bien definidas para cada una de las personas que lideran (DA 1)" y de corresponsabilidad, asumiendo tareas en las que cada miembro de la comunidad puede aportar su conocimiento,

todos los docentes tienen un punto fuerte que pueden compartir y motivar al resto. (JA 1)

... permite y facilita el liderazgo de otros maestros en función de sus aptitudes. Si uno es buenísimo en dinamización de fiestas, por ejemplo, se le permite que coordine en un momento dado un evento escolar. (TC2)

El liderazgo compartido, además, favorece la implicación y compromiso con el centro “... todos nos hemos de sentir importantes y tener claro que todos tenemos cualidades de liderazgo y entre todos podemos hacer grande un proyecto común (CA3)”.

\subsubsection{Colaboración con el entorno}


En relación a este concepto, la información ofrece las categorías emergentes detalladas en el cuadro 6. La colaboración con el entorno es definida mayoritariamente como colaboración con otras entidades educativas para integrar la acción educativa escolar en el marco social y familiar de los alumnos:

Llevar a cabo una acción educativa conjunta y complementaria entre las familias, la escuela y entidades del entorno a partir de unos objetivos comunes compartidos y una actitud basada en la participación, el trabajo y el aprendizaje en red. (TB2)

Hacer una tarea compartida con agentes externos al centro: entidades, familias, servicios externos, ...(JA1)

Cuadro 6. Categorización del concepto de colaboración con el entorno

\begin{tabular}{lc}
\hline \multicolumn{1}{c}{ CATEGORÍAS EMERGENTES } & No DE CITAS \\
\hline Colaboración con entidades educativas & 11 \\
Recurso de aprendizaje & 3 \\
Conocer el contexto & 3 \\
Incorporación en el aula & 2 \\
Toma de decisiones conjunta & 1 \\
Retorno de conocimiento a la sociedad & 1 \\
Responsabilidad socio política & 1 \\
\hline Total de citas & 22 \\
\hline
\end{tabular}

Fuente: Elaboración propia.

Desde esa perspectiva, el entorno se considera como un recurso de aprendizaje, "...el entorno es una fuente imprescindible para un aprendizaje más vivo, funcional y significativo (DB1)", "aprender a partir de las propuestas que nos ofrece el entorno... (CA4)" y la optimización de recursos facilitando el desarrollo de actividades académicas que, sin esa colaboración, sería difícil de realizar “... hacer uso de las instalaciones y espacios del pueblo/ciudad,... (MC1)", "todas aquellas acciones que necesiten de la relación y coordinación con entidades, organismos y personas para realizar actividades fuera del aula y que, por tanto, requieren del entorno (DA 1)”. Así mismo, la colaboración también se ha de dar en sentido recíproco, es decir, la escuela debe ofrecer recursos y actividades a otros colectivos de la comunidad de manera que potencie un retorno del conocimiento a la sociedad

\footnotetext{
...hacer propuestas de colaboración para realizar actividades, talleres, reuniones, conferencias, que enriquezcan a todo el mundo. (CA4)

integrar el entorno cercano en las actividades y asuntos de la escuela de manera que se integre y forme parte de la comunidad educativa. (TC2)
}

Otra línea de interpretación apunta a la necesidad de que tanto el profesorado como el alumnado conozcan y respeten el entorno como componente fundamental para su integración en la comunidad social “...saber dónde estás, qué características socioculturales tiene la escuela (CA2)”, “...que entorno y escuela estén en continuo contacto y que los niños sean conscientes de eso y sean partícipes (MC1)”, “... Ese conocimiento por parte del el profesorado favorece, además, una mayor adecuación de la acción educativa a las necesidades particulares del alumnado “... ser sensible a las necesidades de los alumnos de la escuela y velar por la obtención y/o gestión de ayudas y otros recursos (DB1)". 


\subsection{Impacto de las competencias de coordinación, liderazgo y colaboración con el entorno en el ejercicio docente. Importancia y percepción del estado actual de las mismas}

En este apartado les pedimos que ordenen las tres competencias analizadas según su grado de impacto en el ejercicio docente ( $1=$ mayor impacto, $2=$ impacto medio y $3=$ menor impacto). El gráfico 1 muestra el resultado de las competencias seleccionadas en primera, segunda y tercera opción. Las dos primeras opciones elegidas muestran que, globalmente, la coordinación sería la competencia con mayor impacto, seguida del liderazgo y de la colaboración con el entorno.

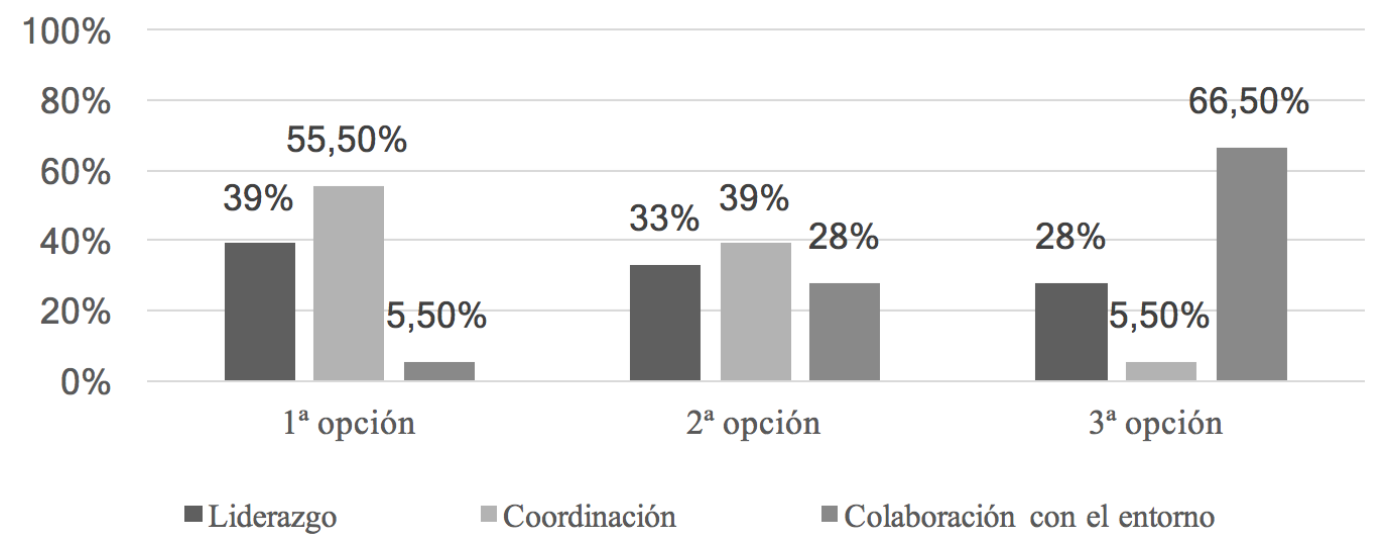

Gráfico 1. Competencias según el impacto en el ejercicio docente Fuente: Elaboración propia.

Si se analizan esos datos en relación a los años de experiencia de los maestros consultados, se observa que los de menos años de experiencia otorgan a la coordinación mucha más importancia que al liderazgo ( $71 \%$ vs $29 \%)$, mientras que los más experimentados otorgan un valor similar a ambas competencias (alrededor del $45 \%$ ).

También se les pide que valoren del o al 10 la importancia que conceden a las competencias de coordinación, liderazgo y colaboración con el entorno, así como el estado de dichas competencias en la profesión docente (en general) y en su propia escuela (en particular) (gráfico 2).

Respecto a la importancia, observamos que las tres competencias han sido altamente valoradas (entre 8,58 y 9,05). En relación al estado de estas competencias en la profesión docente, la opinión general es que deberían mejorarse todas, pero especialmente la coordinación $(5,32)$. El estado de esas competencias en sus respectivas escuelas tiene mejor puntuación (notable), cosa que denota doblemente tanto su reconocimiento positivo como la percepción del margen de mejora existente.

Los maestros y equipos directivos opinan diferente sobre la importancia de esas competencias en función de si han realizado tutorías de prácticas o no:

- Los que han sido tutores, establecen el siguiente orden de importancia: coordinación, colaboración con el entorno y liderazgo.

- Los que no lo han sido, en cambio, conceden la máxima importancia al liderazgo, seguido de la coordinación y de la colaboración con el entorno. 


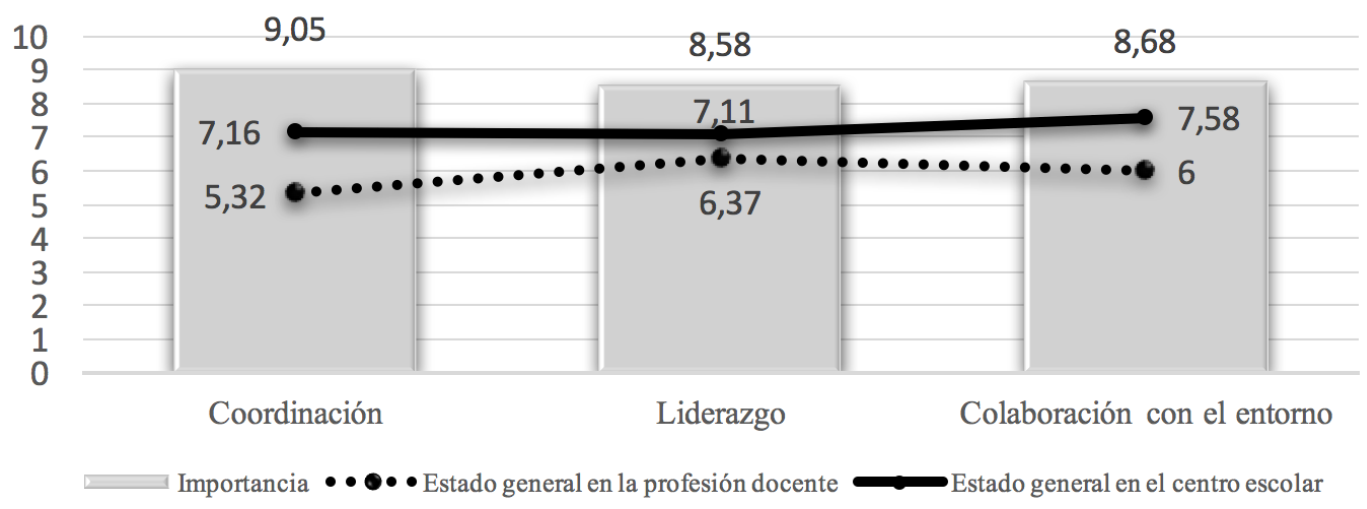

Gráfico 2. Percepción de las competencias en relación a su importancia y estado actual

Fuente: Elaboración propia.

Así mismo, si se analizan estos datos globales en función de algunas variables personales y profesionales, se perciben algunas tendencias. En relación al género, se observa coincidencia entre hombres y mujeres a la hora de considerar la colaboración con el entorno la competencia mejor desarrollada en su propio centro. Sin embargo, discrepan sobre la situación de las otras dos:

- El desarrollo de la competencia del liderazgo en el propio centro es valorado más alto (en segunda posición) por los hombres que por las mujeres (última posición). Dos de los tres directores son hombres.

- En cuanto a la coordinación, son las mujeres quienes la valoran más alto (segunda posición), mientras que es valorada en última posición por los hombres.

Según la edad, los participantes con menos de 40 años consideran la colaboración con el entorno la mejor desarrollada en su propia escuela. Para los de 40 años o más, la más desarrollada sería la coordinación.

\subsection{Percepción de maestros y equipos directivos sobre el nivel competencial del alumnado y del profesorado novel}

En este apartado se pedía a maestros y equipos directivos que valoraran del o al 10 la percepción del nivel competencial con el que llegan al centro escolar los maestros noveles y/o los alumnos en prácticas. Como se observa en el gráfico 3, la competencia más valorada es la responsabilidad en el trabajo (8). En contraposición, la menos valorada es la relación con las entidades del entorno $(5,08)$.

En función de algunas variables analizadas, se han detectado algunas tendencias. Teniendo en cuenta el género, existe unanimidad en valorar la responsabilidad en el trabajo como un punto fuerte de los estudiantes en prácticas y los maestros noveles.

Sin embargo, en otros aspectos los hombres se muestran más críticos y valoran por debajo de 5 las siguientes competencias: resolución de conflictos $(4,50)$, análisis conjunto de situaciones complejas $(4,50)$, impulso y dinamización de grupos de trabajo $(4,67)$, relación con las entidades del entorno $(4,67)$ y gestión del aula $(4,75)$. De éstas, la que obtiene una puntuación más baja por parte de las mujeres es la relación con las entidades del entorno $(5,22)$. 


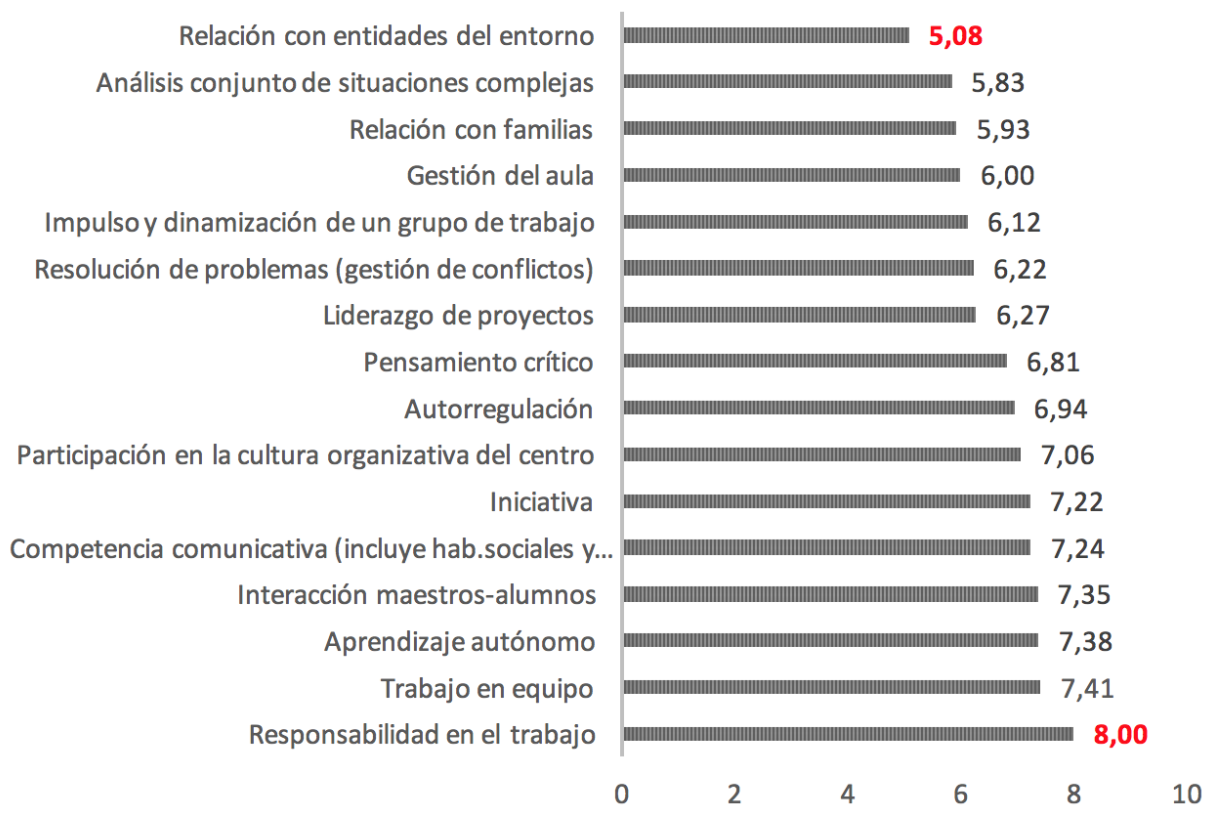

Gráfico 3. Percepción nivel competencial de maestros noveles y alumnado en prácticas Fuente: Elaboración propia.

Finalmente, según la edad, aun coincidiendo en considerar que noveles y practicantes se comportan con responsabilidad en el trabajo, se constata una diferencia clara en la percepción de los profesionales de 40 años o más respecto al liderazgo en proyectos por parte de los recién llegados a la escuela. Éstos consideran que lo tienen menos desarrollado, en contraposición a cómo lo perciben los profesionales de menos de 40 años (5,17 vs. 7,00$)$.

\section{Discusión y conclusiones}

Recientes estudios internacionales apuntan al concepto de teacher agency y enfatizan la autopercepción del maestro como un profesional capaz de llevar a cabo cambios intencionales dirigidos al aumento de la calidad de la educación, tanto en su práctica como en las condiciones con que se encuentra en el ejercicio docente. Además de ciertas cualidades personales, parece necesario que el futuro maestro reciba patrones para desarrollar tal agency a lo largo de su formación inicial: ello dependería en buena parte de haber tenido una formación en que los estudiantes reciban el apoyo y las oportunidades de ser actores críticos de la profesión docente (Biesta, Priestley y Robinson, 2015). Este artículo se basa en una investigación aplicada que pretende mejorar la formación inicial de los estudiantes del Grado de Educación Infantil en relación a la adquisición de competencias relacionadas con el liderazgo pedagógico. En concreto, hemos analizado las opiniones de los equipos directivos y maestros que acogen estudiantes en prácticas en sus centros para conocer desde qué concepción y modelo de liderazgo y también desde qué prácticas realizan lo que podemos llamar primeras experiencias de inserción profesional a la función docente.

Se puede concluir que los maestros y equipos directivos consultados priorizarían la importancia de la coordinación sobre el liderazgo y la colaboración con el entorno para el buen funcionamiento de los centros, al tiempo que perciben como necesarias mejoras 
profesionales en todas ellas. Los docentes más experimentados, sin embargo, equilibran la relevancia de la coordinación y del liderazgo. El valor de la coordinación para un buen ejercicio docente es resaltado desde hace algún tiempo por los estudios sobre el liderazgo distribuido citados anteriormente (Bolívar, 2010, 2011; Danielson, 2006; Leithwood, 2009; Leithwood et al., 2006; Murillo, 2006; OCDE, 2009; Spillane, 2006). Recientemente su importancia se está haciendo más explícita bajo el epígrafe de "liderazgo intermedio" (middle leadership) (Day, Gu, y Sammons, 2016; Gronn, 2003; Grootenboer, EdwardsGroves, y Rönnerman, 2015; Iranzo-García, Tierno-García, Barrios-Arós, 2014; Louis et al., 2010;). Se busca comprender de qué forma el liderazgo distribuido se convierte en acciones coordinadas para que en los centros educativos se ejerza liderazgo también desde los niveles intermedios.

Concretamente y en referencia a las competencias que estamos analizando, en los cuestionarios administrados, la coordinación es conceptuada como necesaria para llegar a acuerdos consensuados en los procesos educativos, evitar incongruencias y construir conocimiento, sobre todo orientado a la gestión del aula. Se concreta en establecer líneas metodológicas comunes a través de encuentros y reuniones regulares, así como de trabajo en equipo. Como apuntan Fairman y Mackenzie (2014), cuando los profesores salen de la propia clase y se comprometen con otros, son más capaces de asumir riesgos y ello favorece el ejercicio de diferentes roles de liderazgo para los que cada uno se siente preparado. La colaboración con la comunidad y el coliderazgo son metas también presentes en la definición que hacen de la coordinación. Sus concepciones se alinean con Perrenoud (2007) quien afirma que el saber participar en la gestión de la escuela y trabajar en equipo son competencias necesarias de la práctica docente y destaca la importancia de la coordinación de los agentes de una institución educativa y también su colaboración con el resto de la comunidad.

La competencia de liderazgo se entiende como el desarrollo de funciones que permitan marcar direcciones y ser referencia, motivar e implicar. Aparece de nuevo el componente de gestión de aula como escenario privilegiado, y se alude a competencias (o características personales) como la comunicación, la negociación, la empatía y la capacidad de resolución de conflictos. Se constata que el liderazgo es visto como una competencia desarrollada en las diferentes situaciones que afronta el docente (aula, proyectos, relación con las familias, gestión, entre otras), aunque se asocia especialmente a la figura del tutor. Se haría, por tanto, un énfasis en el liderazgo pedagógico (Bolívar, 2011; York-Barr y Duke, 2004). Se habla también de corresponsabilidad y de coordinación, en coherencia con el modelo de liderazgo distribuido e intermedio, aunque muchas veces ellos mismos no lo conceptúen como liderazgo.

Colaborar con el entorno se concibe como la competencia de conocer las características y recursos sociales, económicos, culturales, etc. del entorno, así como de tener proyectos conjuntos con servicios o entidades locales y de desarrollarlos dentro y fuera de los centros educativos. Estas concepciones se acercan a la dimensión del liderazgo distribuido y ampliado al "liderazgo de sistema" (Apple y Beane, 1997; Comellas, 2010; Gairín y San Fabián, 2002; Hopkins y Higham, 2007) que completa las de liderazgo pedagógico y distribuido tratadas anteriormente.

Dadas las características de la muestra analizada, no destacaríamos, más allá de las ya mencionadas en el tratamiento cuantitativo, otras grandes diferencias entre las opiniones 
sobre esas competencias (recogidas en las valoraciones cualitativas) respecto a las variables edad, género y experiencia docente y/o de tutorización.

En las tres competencias se evidencia un desequilibrio entre la valoración que los docentes y equipos directivos consultados consideran que debería tener en la profesión docente (entre 8 y 10) y la que perciben (entre 5 y 7 ), por lo que se trataría de competencias a mejorar. Reconocen que en sus centros estas competencias están notablemente desarrolladas, es decir, en mejor estado que en el común de la profesión.

El hecho de que, tal como hemos visto, la docencia está compartida con tareas de coordinación de una manera muy generalizada, unido a que estamos ante un colectivo de docentes experimentado y bastante estable desde el punto de vista laboral y profesional, puede explicar esta valoración, ya que pueden plantearse aspectos de mejora escolar que incluyan, en este caso, la ampliación del liderazgo a un mayor rango de instancias educativas.

Sobre la formación inicial, los encuestados consideran mejorables las competencias de los estudiantes en la resolución de conflictos, análisis de situaciones complejas, gestión del aula y relación con familias y entidades del entorno, y señalan adquiridas las de trabajo en equipo, iniciativa, responsabilidad, aprendizaje autónomo, participación en la cultura del centro e interacción con los alumnos.

Como se ve, las cuatro competencias de liderazgo educativo esenciales que Álvarez (2010) destacaba, a saber, competencia de pensamiento estratégico, de gestión del aprendizaje, de relación con las personas y competencia para la creación y animación de estructuras organizativas, siguen siendo el foco de la formación inicial y de la formación permanente del profesorado y la mejora escolar. Esa misma formación inicial ha de abordar la identidad de liderazgo de los estudiantes, en su gran mayoría mujeres, para atender al fenómeno de masculinización de los roles de dirección de centros todavía presente en nuestros contextos, español y europeo (Eurydice, 2013). En esa misma línea, estamos de acuerdo con que es necesario atender a los indicios de ciertas diferencias en los modos de dirigir cuando las directoras son mujeres a favor de una mayor tendencia a desplegar liderazgos pedagógico y distribuido (mayor comunicación en torno a la misión de la escuela, alto foco en la coordinación para mejorar la instrucción y creación de climas escolares para el aprendizaje positivos) para aumentar su presencia en los puestos de dirección escolar (Hallinger, Dongyu y Wang, 2016).

La colaboración con las escuelas para incrementar la consecución de competencias docentes de liderazgo, coordinación y relación con el entorno se muestra como un espacio privilegiado y disponible para la formación inicial de maestros. En ese mismo sentido, el período de prácticas en las escuelas se percibe como oportuno e idóneo para el desarrollo de estas competencias junto a los docentes y, sobre todo, junto a tutores y agentes implicados en el prácticum en múltiples situaciones profesionales.

Tenemos en cuenta los límites epistemológicos asociados a la propia metodología de estudio de caso que permite profundizar, pero no realizar generalizaciones. Así, nos proponemos seguir analizando las tendencias recogidas y contrastarlas con las perspectivas de los propios agentes participantes y con la revisión teórica del ámbito de la dirección escolar.

Somos conscientes de que en algunos análisis hemos sumado y no diferenciado las percepciones que tienen los maestros y equipos directivos sobre el desarrollo competencial 
de noveles y estudiantes de prácticas (de últimos cursos). El motivo es centrarnos en la preparación de docentes y futuros docentes a los que no les separa mucha distancia en el tiempo, cosa que podría informar sobre la preparación atribuida más a la formación inicial que a la permanente. Así mismo, se presenta de forma indiferenciada la visión de los docentes y de los equipos directivos ya que provienen sólo de tres escuelas, aun sabiendo que puede ser muy diversa. Ese análisis diferenciado es objeto también de nuestra investigación.

Finalmente, la información debe contextualizarse en relación al conjunto de los objetivos de la investigación, globalmente entendida. En la misma, y a partir de una fase diagnóstica, se diseñaron y se están desarrollando y evaluando acciones formativas dirigidas a estudiantes, profesorado universitario y maestros en ejercicio, que pretenden consolidar "planes de acogida" para que las escuelas promuevan desarrollo competencial sobre esas mismas competencias tanto para los equipos directivos como para el conjunto del profesorado (incluido el profesorado novel o de nueva incorporación en el centro) y tutores y estudiantes en prácticas.

Vemos necesario contrastar las opiniones de equipos directivos, maestros, estudiantes y profesorado universitario ampliando la investigación a un mayor número de centros educativos, así como a diversos ámbitos geográficos con particulares recorridos en la definición del liderazgo escolar.

\section{Agradecimientos}

Este artículo ha sido desarrollado en el marco del Proyecto ARMIF 2015-00041 "Competencias de coordinación, liderazgo compartido y trabajo en red con la comunidad para el desarrollo de la profesión docente y la mejora escolar". Programa de Mejora e Innovación en la Formación de Maestros. Financiado por AGAUR-Agencia de Gestión de Ayudas Universitarias y de Investigación de Cataluña.

\section{Referencias}

Agudo, N. (2017). Formación de formadores para la preparación de profesionales en contextos laborales. Tesis doctoral. Universidad de Granada, España.

Álvarez, M. (2010). Liderazgo compartido: Buenas prácticas de dirección escolar. Madrid: Wolters Kluwer.

Apple, M. W. y Beane, J. A. (Coords.). (1997). Escuelas democráticas. Madrid: Morata.

Aramendi, P., Teixidó, J. y Bernal, J. L. (2010). El acceso a la dirección escolar en los centros públicos del País Vasco. Revista Española de Pedagogía, 246, 313-332.

AQU Catalunya. (2015a). Ocupabilitat $i$ competències dels graduats recents: l'opinió d'empreses $i$ institucions. Principals resultats de l'estudi d'ocupadors 2014. Barcelona: AQU Catalunya.

AQU Catalunya. (2015b). Ocupabilitat $i$ competències dels nous docents. L'opinió dels centres educatius d'educació infantil, primària i secundària. Barcelona: AQU Catalunya.

Barrios-Arós, C., Iranzo-García, P. y Tierno-García, J. M. (2013). Avances teórico-prácticos y legislativos en la profesionalización de la dirección escolar en España. El caso de Cataluña. Profesorado. Currículum y Formación del Profesorado, 17(3), 371-387. 
Biesta, G., Priestley, M. y Robinson, S. (2015). The role of beliefs in teacher agency. Teachers and Teaching. Theory and Practice, 21(6), 624-640. https://doi.org/10.1080/13540602.2015.1044325

Bolívar, A. (2010) ¿Cómo un liderazgo pedagógico y distribuido mejora los logros académicos? Magis, Revista Internacional de Investigación en Educación, 3(5), 79-106.

Bolívar, A. (2011). Aprender a liderar líderes. Competencias para un liderazgo directivo que promueva el liderazgo docente. Educar, 472, 253-275. https://doi.org/10.5565/rev/educar.50

Cano, E. (2011). Buenas prácticas en la evaluación de competencias. Barcelona: Laertes.

Cano, E. (2013). El impacto de la evaluación educativa en el desarrollo de competencias en la universidad. La perspectiva de las primeras promociones de graduados. Referencia: EDU2012-32766. Madrid: MINECO.

Cantón, I. y Arias, A. R. (2008). La dirección y el liderazgo: Aceptación, conflicto y calidad. Revista de Educación, 345, 229-254.

Comellas, M. J. (2010). El treball en xarxa: Un model de recerca i acció participativa per promoure la cooperació de les famílies. Educar, 45, 117-129.

Danielson, C. (2006). Teacher leadership that strengthens professional practice. Alexandria, VA: ASCD.

Day, C., Gu, Q. y Sammons, P. (2016). The impact of leadership on student outcomes: How successful school leaders use transformational and instructional strategies to make a difference. Educational Administration Quarterly, 52(2), 221-258. https://doi.org/10.1177/0013161X15616863

Eurydice. (2013). Key data on teachers and school leaders in Europe. Eurydice report. Luxemburgo: Publications Office of the European Union.

Fairman, J. C. y Mackenzie, S. V. (2014). How teacher leaders influence others and understand their leadership. International Journal of Leadership in Education: Theory and Practice, 18(1), 61-87. https://doi.org/10.1080/13603124.2014.904002

Gairín, J. y Castro, D. (2010). Situación actual de la dirección y gestión de los centros de enseñanza obligatoria en España. Revista Española de Pedagogía, 247, 401-416.

Gairín, J. y San Fabián, J. L. (2002). La participación social en educación. En B. Jiménez y R. Mejías (Coords.), Formación profesional. Orientaciones y recursos (pp. 157-188). Barcelona: Praxis.

Generalitat de Catalunya. (2014). Resolución ENS/906/2014 por la cual se determinan los centros educativos y los puestos de trabajo docentes que tienen la consideración de dificultad especial. Recuperado de http://legislacion.derecho.com/resolucio-ens-0906-2014-23-abril-2014departament-d-ensenyament-5643183

Gronn, P. (2003). The new work of educational leaders: Changing leadership practices in an era of school reform. Londres: Paul Chapman.

Grootenboer, P., Edwards-Groves, C. y Rönnerman, K. (2015). Leading practice development: Voices from the middle. Professional Development in Education, 41(3), 79-93. https://doi.org/10.1080/19415257.2014.924985

Hargreaves, A. (1996). Profesorado, cultura y postmodernidad. Cambian los tiempos, cambia el profesorado. Madrid: Morata.

Hallinger, P., Dongyu, L. y Wang, W. (2016). Gender differences in instructional leadership: A meta-analytic review of studies using the principal instructional management rating scale. 
P. Iranzo-García, M. Camarero-Figuerola, C. Barrios-Arós, J. M. Tierno-García y S. GilabertMedina

Educational Administration Quarterly, 567-601. https://doi.org/10.1177/0013161X16638430

Hopkins, D. y Higham, R. (2007). System leadership: Mapping the landscape. School Leadership and Management, 27(2), 147-166. https://doi.org/10.1080/13632430701237289

Iranzo-García, P., Tierno-García, J. M. y Barrios-Arós, C. (2014). Autoevaluación institucional y dirección de centros inclusivos. Teoría de la Educación. Revista Interuniversitaria, 26(2), 229257. https://doi.org/10.14201/teoredu2014261229257

Iranzo-García, P., Camarero-Figuerola, M., Barrios-Arós, C. y Tierno-García, J. M. (2018). Formación para la función directiva en la escuela: El caso de Tarragona (Cataluña). Bordón. Revista de Pedagogía. 7O(2), 57-72. https://doi.org/10.13042/Bordon.2018.54487

Leithwood, K. (2009). ¿Cómo liderar nuestras escuelas? Aportes desde la investigación. Santiago de Chile: Fundación Chile.

Leithwood, K., Day, C., Sammons, P., Harris, A. y Hopkins, D. (2006). Seven strong claims about successful school leadership. Nottingham: National College for School Leadership.

León, M. J., Crisol, E. y Moreno, R. (2018). Las tareas del líder inclusivo en centros educativos de zonas desfavorecidas y favorecidas. REICE. Revista Iberoamericana sobre Calidad, Eficacia y Cambio en Educación, 16(2), 21-40. https://doi.org/10.15366/reice2018.16.2.002

Louis, K.S., Leithwood, K., Wahlstrom, K., Anderson, S., Michlin, M., Mascall, B., et al. (2010). Learning from leadership: Investigating the links to improved student learning. http://www.wallacefoundation.org/knowledge-center/Documents/Investigating-theLinks-to-Improved-Student-Learning.pdf

Molina, E. (2004). La mejora del prácticum, esfuerzos de colaboración. Profesorado: Revista de Currículum y Formación del Profesorado, 8(2), 1-29.

Murillo, F. J. (2006). Una dirección escolar para el cambio: Del liderazgo transformacional al liderazgo distribuido. REICE. Revista Iberoamericana sobre Calidad, Eficacia y Cambio en Educación, 4(4e), 11-24.

Negrillo, C. e Iranzo-García, P. (2009). Formación para la inserción profesional del profesorado novel de educación infantil, educación primaria y educación secundaria: Hacia la reflexión desde la inducción y el soporte emocional. Profesorado. Currículum y Formación del Profesorado, 13(1), 158-162.

OCDE. (2009). Mejora el liderazgo escolar. Herramientas de trabajo. París: OCDE.

Perrenoud, P. (2007). Desarrollar la práctica reflexiva en el oficio de enseñar. Profesionalización y razón pedagógica. Ciudad de México: Colofón.

Putnam, R. y Borko, H. (2000). El aprendizaje del profesor: Implicaciones de las nuevas perspectivas de la cognición. En B. Biddle, T. Good y L. Goodson (Eds.), La enseñanza y los profesores. La profesión de enseñar (pp. 219-309). Barcelona: Paidós.

Spillane, J. P. (2006). Distributed leadership. San Francisco, CA: Jossey-Bass

Tierno-García, J. M., Iranzo-García, P. y Barrios-Arós, C. (2013). El compromiso organizativo e institucional para enseñar y evaluar competencias en la universidad. Revista de Educación, 361, 223-251.

York-Barr, J. y Duke, K. (2004). What do we know about teacher leadership? Findings from two decades of scholarship. Review of Educational Research, 74(3), 255-316. https://doi.org/10.3102/00346543074003255 


\section{Breve CV de las autoras}

\section{Pilar Iranzo-García}

Profesora del Departamento de Pedagogía de la Universidad Rovira i Virgili (URV) en Comisión de Servicios (Acreditada Titular ANECA, 2011). Licenciada y doctora en Pedagogía y diplomada en Profesorado. Imparte docencia en los grados de maestro y pedagogía y en el máster de formación del profesorado de ESO, bachillerato, formación profesional y enseñanza de idiomas, en materias vinculadas a la mejora escolar y el asesoramiento a profesionales e instituciones educativas. Sus líneas de investigación son: mejora y liderazgo escolares, formación y desarrollo profesional del profesorado y docencia universitaria. ORCID ID: https://orcid.org/o000-0002-8516-9205. Email: pilar.iranzo@urv.cat

\section{Marta Camarero-Figuerola}

Profesora e Investigadora Postdoctoral en el Departamento de Pedagogía de la Universitat Rovira i Virgili. Licenciada en Pedagogía por la Universitat de Barcelona en el año 2011. Premio Extraordinario del Máster Formación de Formadores por la Universitat Rovira i Virgili en el 2013 y Doctora en Pedagogia por la misma universidad en el año 2015. En la actualidad es Imparte docencia en los Grados de Educación Infantil y Primaria; Educación Social y Pedagogía. Sus líneas de investigación son entre otras, el liderazgo educativo, docencia universitaria y mejora escolar. ORCID ID: https://orcid.org/0000-0001-6116-0882. Email: marta.camarero@urv.cat

\section{Charo Barrios-Arós}

Profesora titular del Departamento de Pedagogía de la Universidad Rovira i Virgili (URV). Licenciada y doctora en Pedagogía. Ha sido directora del Departamento de Pedagogía de la URV durante ocho años. Imparte docencia en materias vinculadas a la planificación y organización educativa. Las líneas de investigación son: formación y desarrollo profesional del profesorado, docencia universitaria y mejora escolar y dirección de centros. ORCID ID: https://orcid.org/0000-0002-3620-9338. Email: charo.barrios@urv.cat

\section{Juana-María Tierno-García}

Profesora contratada doctora del Departamento de Pedagogía la Universidad Rovira i Virgili (URV). Licenciada y doctora en Pedagogía. Máster Interuniversitario de Tecnología Educativa. Directora del Departamento de Pedagogía de la URV. Imparte docencia en grado en materias relacionadas con la investigación y la evaluación en educación. Las líneas de investigación son: formación del profesorado, docencia universitaria y mejora escolar y dirección de centros. ORCID ID: https://orcid.org/oooo0002-1027-7332. Email: juanamaria.tierno@urv.cat

\section{Sandra Gilabert-Medina}

Profesora Lectora del Departamento de Pedagogía de la Universidad Rovira i Virgili (URV). Diplomada en Magisterio y Licenciada en Psicopedagogía por la URV. Doctora en Psicología de la Educación por la Universidad de Barcelona. Responsable del Grado de Educación Infantil de la Sede Baix Penedès de la URV y Directora del Master en 
P. Iranzo-García, M. Camarero-Figuerola, C. Barrios-Arós, J. M. Tierno-García y S. GilabertMedina

Educación Sistémica Multidimensional (título propio) de la URV. Imparte docencia en el Grado de Ed. Infantil en asignaturas relacionadas con la didáctica. Las líneas de investigación son: Psicomotricidad y aprendizaje, argumentación en el aula, enseñanzaaprendizaje de las matemáticas y ciencias naturales. ORCID ID: https://orcid.org/oooo0003-1003-0599. Email: sandra.gilabert@urv.cat 